\title{
Signaling through estrogen receptors modulates telomerase activity in human prostate cancer
}

\author{
Simona Nanni, ${ }^{1,2}$ Michela Narducci, ${ }^{1}$ Linda Della Pietra, ${ }^{1,3}$ Fabiola Moretti, ${ }^{1,4}$ \\ Annalisa Grasselli, ${ }^{1,4}$ Piero De Carli, ${ }^{1}$ Ada Sacchi,${ }^{1}$ Alfredo Pontecorvi, ${ }^{1,2}$ \\ and Antonella Farsetti ${ }^{1,4}$
${ }^{1}$ Molecular Oncogenesis Laboratory and Urology Division, Regina Elena Cancer Institute, Experimental Research Center, Rome, Italy
${ }^{2}$ Institute of Medical Pathology, Catholic University, Rome, Italy
${ }^{3}$ University of Rome, La Sapienza, Rome, Italy
${ }^{4}$ Institute of Neurobiology and Molecular Medicine, National Research Council, Rome, Italy

\begin{abstract}
Sex steroid hormone receptors play a central role in all stages of prostate cancer. Here, we tested whether estrogen receptor (ER) signaling contributes to telomerase activation, an early event in prostate tumorigenesis. Following 17ß-estradiol $\left(\mathrm{E}_{2}\right)$ treatment, both mRNA encoding the catalytic subunit of human telomerase (hTERT) and telomerase activity were promptly induced in human prostate normal epithelial cells, fresh explants from benign prostate hyperplasia, and prostate cancer explants and cell lines. Reporter expression studies and in vivo chromatin immunoprecipitation assays revealed $\mathrm{E}_{2}$-dependent $b T E R T$ promoter induction and showed that both ER $\alpha$ and ER $\beta$ bound this sequence. Crucially, addition of the anti-estrogen 4-hydroxytamoxifen caused a differential recruitment in vivo of ER $\alpha$ and ER $\beta$ onto the $b T E R T$ promoter and inhibited telomerase activity. Treatment with the aromatase inhibitor letrozole, which prevented testosterone-mediated interaction between ER and the $h T E R T$ estrogen response element, resulted in a negative regulation of telomerase activity. Thus, intracellular conversion of androgens to estrogens may contribute to the etiopathogenesis of prostate cancer. Given the present evidence for direct control of $b T E R T$ gene expression and telomerase activity in the prostate by the ER, we suggest that this transcriptional regulator represents a possible therapeutic target in prostate cancer.
\end{abstract}

J. Clin. Invest. 110:219-227 (2002). doi:10.1172/JCI200215552.

\section{Introduction}

Strong experimental evidence has linked the maintenance of functional telomeres, and thus telomerase activity, to human cancer $(1,2)$. The absence of the enzyme in human somatic cells and their replicative senescence are considered a tumor suppressor mechanism, while activation of telomerase has been identified as an essential requirement for unlimited cell proliferation (3-5). To date, telomerase has been detected in over $95 \%$ of tumor samples and is there-

Received for publication March 28, 2002, and accepted in revised form June 5, 2002.

Address correspondence to: Antonella Farsetti, Molecular Oncogenesis Laboratory, Regina Elena Cancer Institute, Via delle Messi D'Oro 156, 00158 Rome, Italy. Phone: (39-06) 5266-2531; Fax: (39-06) 4180526; E-mail: farsetti@ifo.it.

Conflict of interest: No conflict of interest has been declared. Nonstandard abbreviations used: prostate cancer (PCa); prostate-specific antigen (PSA); estrogen receptor (ER); androgen receptor (AR); ER subtype $\beta$ (ER- $\beta$ ); $17 \beta$-estradiol $\left(\mathrm{E}_{2}\right)$; human telomerase (hTERT); triiodothyronine $\left(\mathrm{T}_{3}\right)$; testosterone $(\mathrm{T})$; 4-hydroxytamoxifen (OHT); prostate epithelial cells (PrECs); telomere repeat amplification protocol (TRAP); benign prostate hyperplasia (BPH); estrogen response element (ERE); vitellogenin promoter (VIT); chromatin-cross-linked immunoprecipitation (ChIP); selective ER modulators (SERMs) catalytic subunit of human telomerase (hTERT); estrogen receptor subtype $\alpha$ (ER $\alpha$ ); cyclin B1 (Cyc B1). fore one of the most widespread tumor markers (6). In prostate cancer $(\mathrm{PCa})$, the most common malignancy in elderly men in Western countries (7), increased telomerase activity is already evident at the very early stages of the disease, namely prostate in situ neoplasia. Indeed, evaluation of telomerase activity in prostate biopsies has become a valuable diagnostic marker for this malignancy $(8,9)$ in addition to measurements of increased serum levels of prostate-specific antigen (PSA). The molecular mechanisms underlying telomerase activation during cancer development are still largely undefined. Recently, we provided evidence that estrogens can reverse telomerase silencing in normal telomerase-negative ovary epithelial cells by transcriptional activation of the catalytic subunit of the enzyme (10) whose induction is a rate-limiting step for telomerase activity. The identification of the catalytic subunit of human telomerase (hTERT) as a target of ligand-activated estrogen receptor (ER) has prompted us to investigate whether such a mechanism could underlie telomerase activation in hormone-dependent tumors. We focused on the prostate gland for a number of reasons. First, a distinguishing feature of $\mathrm{PCa}$ is its intimate association with aging, the most significant risk factor for this malignancy (7). Second, due to their in vivo selfrenewal and, consequently, their short telomeres, 
prostate epithelial cells from which adenocarcinomas arise may be particularly sensitive to variations in telomere length. Third, steroid hormone receptor signaling affects initiation and progression of PCa. Although the relevance of androgen receptor (AR) in the development of prostate and in the evolution of PCa is well established (7), it does not account for the frequent failure of conventional androgen-deprivation therapy in advanced disease.

Several recent reports have focused on the role of ERs in normal and transformed prostate epithelium (11-15). It is becoming evident that the decline of circulating androgens, resulting in a characteristic agerelated decrease of the androgens-to-estrogens ratio, is a contributing factor in PCa development $(7,16)$. A direct action of estrogens on the growth of normal and malignant prostate cells was originally proposed (17), and recent literature suggests that estrogens may exert these direct effects via their own receptors $(12,13,18,19)$. Interestingly, the newly discovered ER subtype $\beta$ (ER- $\beta$ ) is abundantly expressed in the epithelial compartment, whereas the $\alpha$ subtype (ER- $\alpha$ ) is mostly present in the stromal compartment in both the rat and human prostate glands $(7,14,20)$. The relative expression levels of ERs in prostate adenocarcinoma are still quite controversial. ER- $\beta$ mRNA has been detected in highly enriched or pure human prostate epithelial cells and in PCa cell lines $(12,20)$, whereas the ER- $\alpha$ gene appears to be largely inactivated by DNA methylation in most PCa cells and PCa specimens $(12,13)$. Recent reports, however, showed a frequent loss of ER- $\beta$ expression in PCa samples relative to normal prostate tissue in both clinically localized and hormone-refractory tumors $(14,21-23)$. Despite the controversy concerning the expression of the two ER subtypes in normal and malignant prostate epithelium, a growing body of evidence suggests that abnormalities in ER signaling may contribute to the complex molecular pathogenesis of PCa $(11,24)$.

The aim of the present study was to investigate whether ER signaling may be responsible for telomerase activation in prostate epithelial cells, thus representing a pathogenetic factor in prostate carcinogenesis.

\section{Methods}

Hormones and inhibitors. Hormones and inhibitors used were as follows: $17 \beta$-estradiol $\left(\mathrm{E}_{2}\right)$, triiodothyronine $\left(\mathrm{T}_{3}\right)$, testosterone $(\mathrm{T})$, 4-hydroxytamoxifen $(\mathrm{OHT})$ (Sigma-Aldrich, Milan, Italy), methyltrienolone (R1881; gift from A. Banhiamad, Justus Liebig University, Gissen, Germany), and letrozole (gift from M. Maggi, University of Florence, Florence, Italy).

$A b$ 's. Ab's used were as follows: $\alpha$ ER- $\alpha$ : HC-20, C-311, D-12 (Santa Cruz Biotechnology Inc., Santa Cruz, California, USA), Ab-1 (NeoMarkers Inc., Fremont, California, USA), and H-222 (gift from G.L. Greene, Ben May Institute, University of Chicago, Chicago, Illinois, USA; $\alpha$ ER- $\beta$ : L-20, H-150 (Santa-Cruz Biotechnology
Inc.), and 210-180-C050 (Alexis Biochemicals, Läufelfingen, Switzerland); $\alpha$ NF-Y: pR $\alpha$ YB (gift from R. Mantovani, University of Modena and Reggio, Modena, Italy); $\alpha$ AR: C-19 (Santa-Cruz Biotechnology Inc.).

Isolation and establishment of prostate epithelial cells. Primary cultures of prostate epithelial cells were explanted from fresh tissue specimens and characterized for the expression of PSA by direct staining with a specific $\mathrm{Ab}(25)$. The histological diagnosis and Gleason score was determined in each case during a routine clinical workup after surgery. After approval by the Ethic Committee of the Regina Elena Cancer Institute in Rome, tumor specimens were obtained from three clinically localized prostate carcinoma and two benign prostate hyperplasia.

Cell lines. Prostate epithelial cells (PrEC) were cultured according to the manufacturer's protocols (Clonetics Bio-Whittaker, Milan, Italy). Human PCa cell lines (gift from G. Sica, Catholic University, Rome, Italy) were cultured as follows: LNCaP in RPMI-1640 supplemented with $10 \% \mathrm{FBS}, 4.5 \mathrm{~g} / \mathrm{l}$ glucose, and $0.1 \mathrm{M}$ HEPES; PC-3 in F12 Coon's modified medium supplemented with $10 \%$ FBS; and DU145 in RPMI-1640 supplemented with $10 \%$ FBS. At least 72 hours prior to experimental use, the cells were switched to medium supplemented with hormone-deprived serum (26).

RT-PCR analysis. The hTERT and hTR mRNA analysis was carried out by PCR amplification using primers and conditions as described (27). The housekeeping aldolase mRNA, used as external standard, was amplified from the same cDNA reaction using specific primers (10). The intensity of each band was evaluated by densitometry using the NIH Image J 1.24 software (National Institutes of Health, Bethesda, Maryland, USA).

Telomerase assay. Cell extracts were prepared by detergent lysis, and enzymatic activity was detected by the PCR-based telomere repeat amplification protocol (TRAP) (28). All assays were repeated at least three times with three different preparations of cell lysates. Quantitative analysis was performed with the NIH Image 1.61 software. Telomerase activity was quantified by measuring the signals of telomerase ladder bands, and the relative telomerase activity was calculated as a ratio to the internal standard.

Plasmid. Plasmids p3996, p1009, and p1009Mut have been described (10). Expression vectors encoding the human ER- $\alpha$ and ER- $\beta$ were gifts from P. Chambon (Institute de Genetique et de Biologie Moleculaire et Cellulaire, CNRS/INSERM, Illkirch, France) and J.A. Gustafsson (Karolinska Institute, Huddinge, Sweden), respectively.

Formaldebyde cross-linking and immunoprecipitation of chromatin. Exponentially growing cells were crosslinked as described (29). The chromatin solution was precleared by addition of protein- $\mathrm{G}$ (Pierce Chemical Co., Rockford, Illinois, USA) for 1 hour at $4^{\circ} \mathrm{C}$ and incubated with $2 \mu \mathrm{g}$ of specific Ab's overnight at $4^{\circ} \mathrm{C}$, with mild shaking. DNA fragments were recovered and analyzed by PCR $(0.5-1 \mu \mathrm{l}$ of immunoprecipitated 

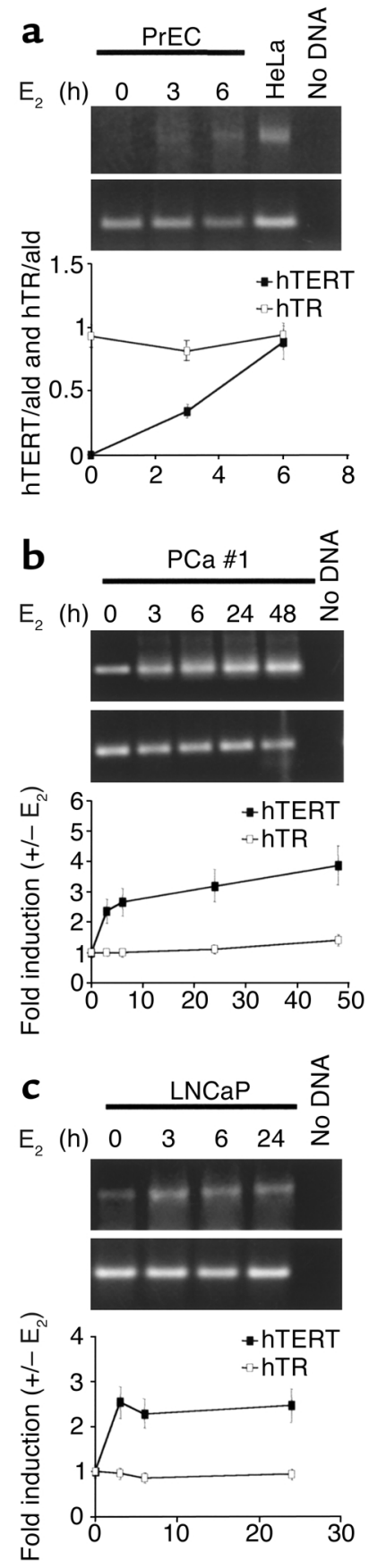
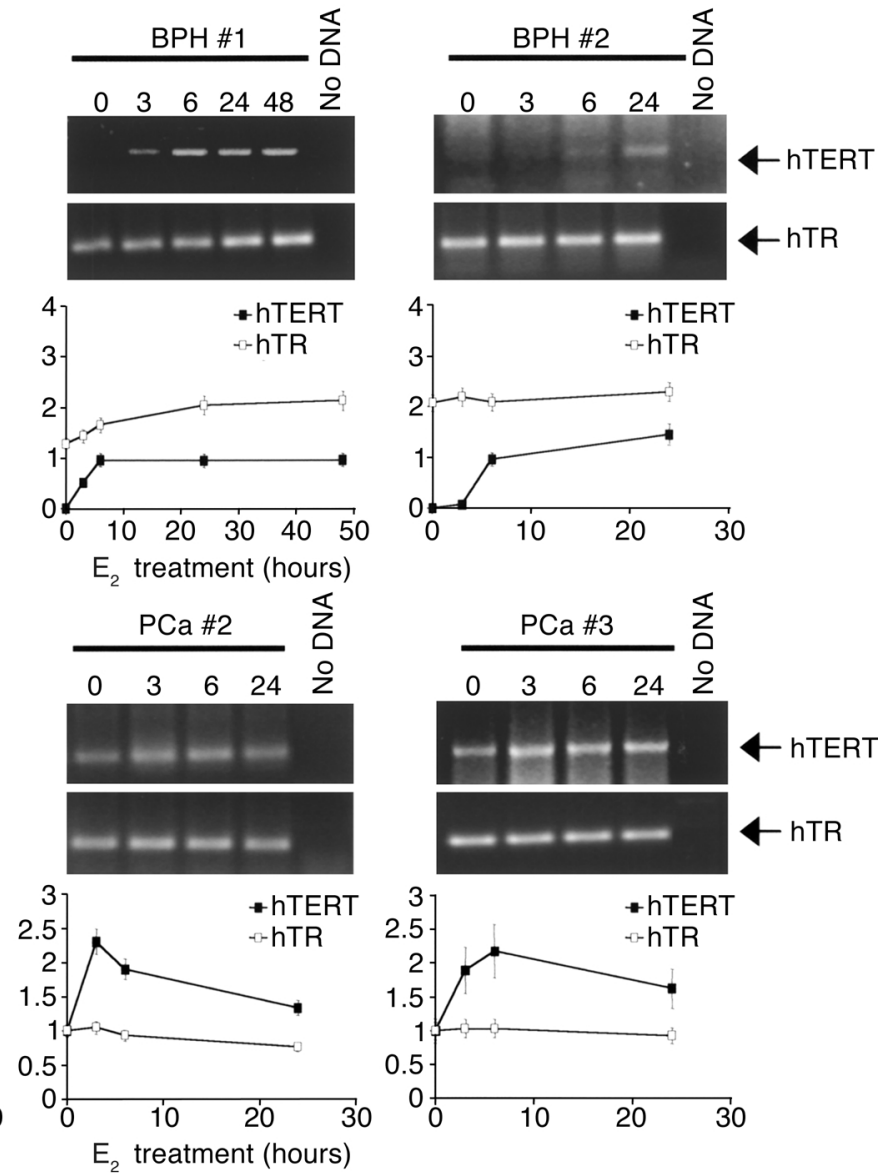

hTR
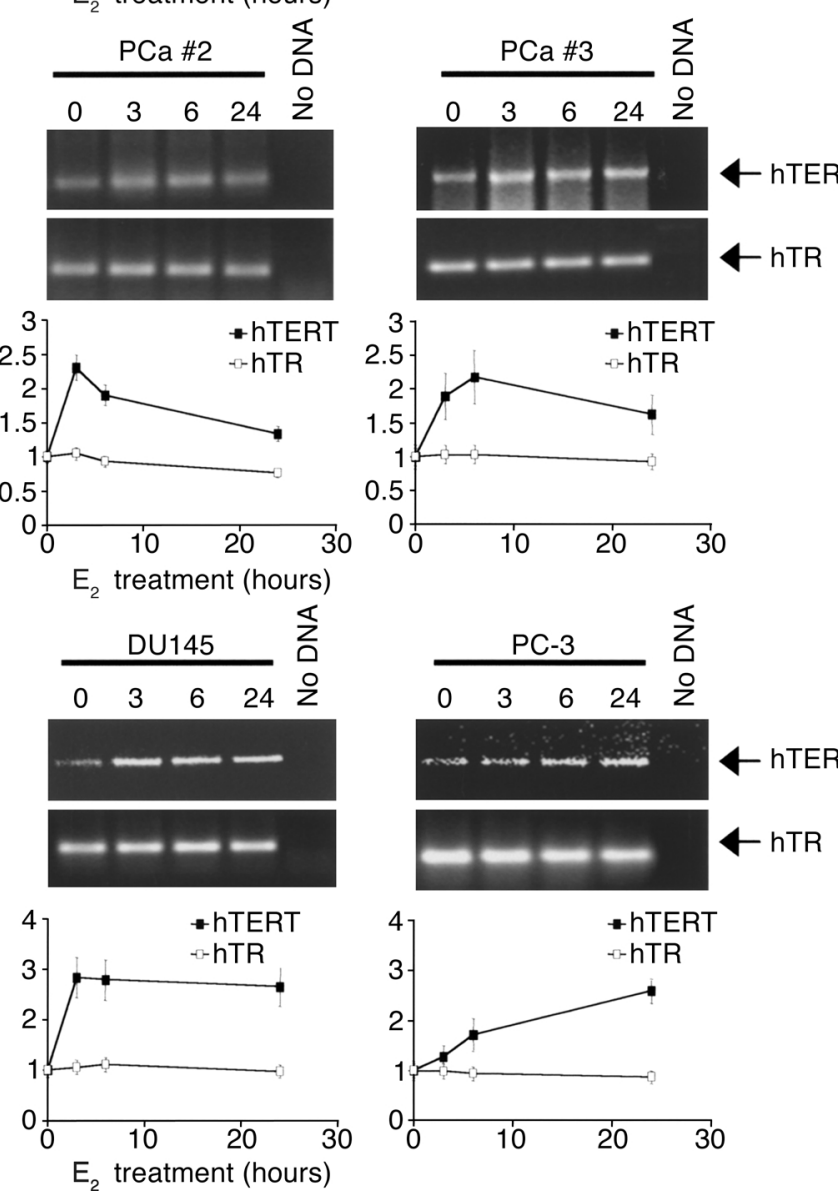

\section{Figure 1}

Induction of hTERT mRNA by $\mathrm{E}_{2}$ in normal and transformed human prostate epithelial cells. Total RNA was extracted from cells cultured in the presence or absence of $E_{2}\left(10^{-7} \mathrm{M}\right)$ for the indicated times. Semiquantitative RT-PCR analysis of hTERT and hTR mRNA levels was performed in (a) normal PrECs and fresh explants from two $\mathrm{BPH}$ lesions (no. 1 and 2), (b) three PCa specimens (no. 1, 2, and 3), and (c) three $\mathrm{PCa}$ cell lines (LNCaP, DU145, and PC-3). HeLa cell RNA (HeLa) and the "no cDNA template" (no DNA) were used as positive and negative controls, respectively. Densitometric analysis of hTERT and hTR mRNA levels in the presence or absence of $E_{2}$ was performed after normalization to values obtained under the same conditions with the control housekeeping gene aldolase (ald). The average values from three independent experiments were expressed as the ratio of hTERT and hTR versus aldolase (hTERT/ald and hTR/ald) in a or as fold of induction $\left(+/-E_{2}\right)$ of normalized values ( $h$ TERT/ald and hTR/ald) in b and $\mathbf{c}$. chromatin) using the following oligonucleotides: hTERT-up 5'-GGC CCC AGG TCT GGA GGG GAC-3'; hTERT-down $5^{\prime}$-ACA GGT GCG TGC GGC GAC CCT TTG$3^{\prime}$. PCR amplification for the cyclin B1 (Cyc B1) promoter was carried out from the same DNA immunoprecipitation as described (30). The same number of PCR cycles (32-35) was used for all samples.

\section{Results}

hTERT expression and telomerase activity in normal and transformed prostate epithelial cells after $E_{2}$ treatment. The catalytic subunit of hTERT has been identified as a transcriptional target of ER- $\alpha$ in normal and transformed epithelial cells from the human ovary (10) and breast (31). Based on these observations, we have investigated whether treatment with $\mathrm{E}_{2}$ affects $h T E R T$ gene expression and telomerase activity in normal prostate epithelial cells, in PCa cell lines, and in fresh explants from lesions representing different stages of disease. Levels of hTERT mRNA in $\mathrm{E}_{2}$-treated and -untreated cells were evaluated by RT-PCR, and the extent of induction was measured by densitometry (Figure 1). As expected, in the unstimulated state hTERT mRNA was undetectable in normal human PrEC cells and in fresh explants from benign prostate hyperplasia (BPH). Treatment with $\mathrm{E}_{2}$ rapidly (between 3 and 6 hours) induced hTERT mRNA 


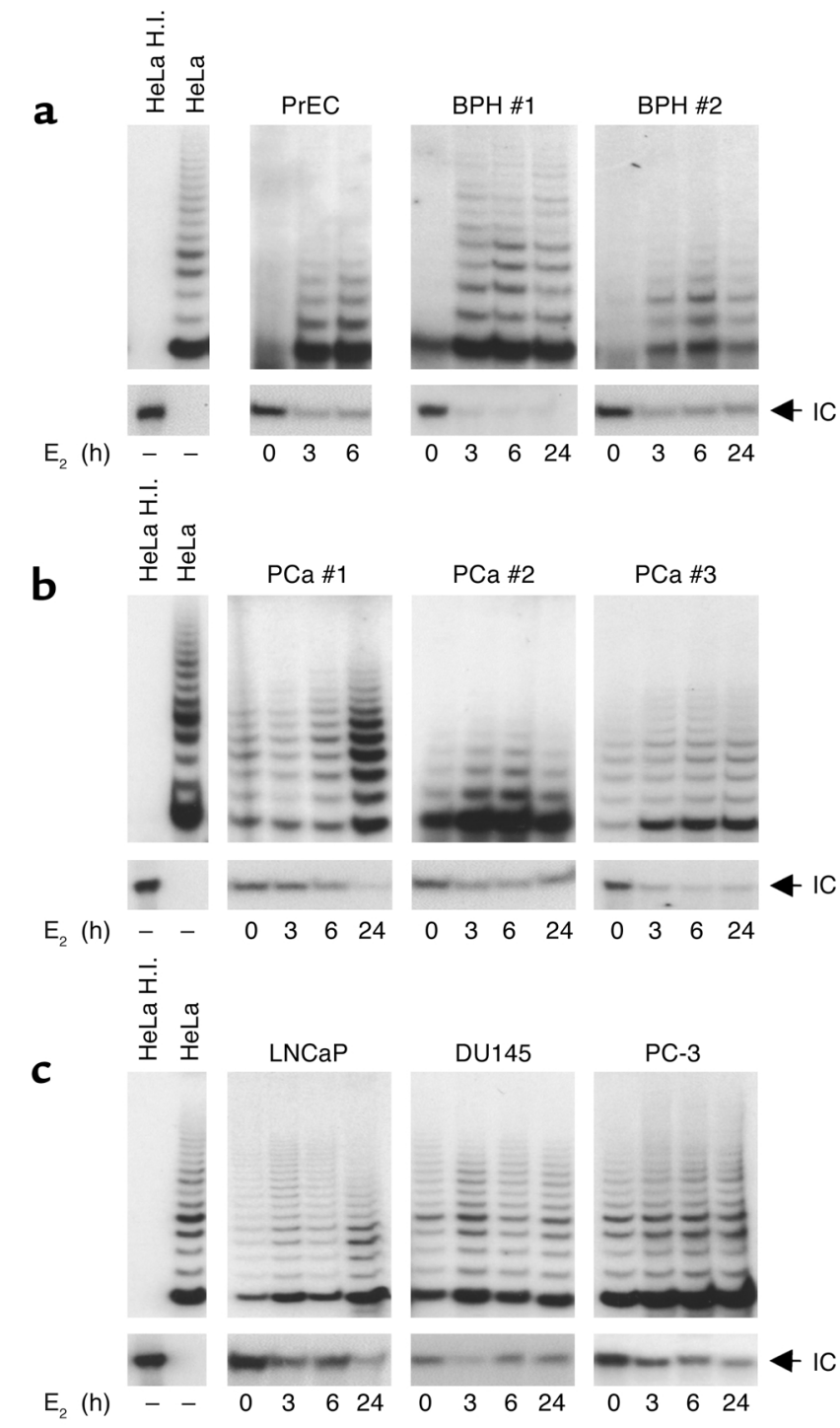

\section{Figure 2}

Induction of telomerase activity by $E_{2}$ in normal and transformed human prostate epithelial cells. Cell extracts from (a) normal PrECs (1 $\mu \mathrm{g})$ and fresh explants from two BPH lesions ( $1 \mu \mathrm{g}),(\mathbf{b})$ three PCa specimens $(0.2 \mu \mathrm{g})$, and (c) PCa cell lines LNCaP, DU145, and PC-3 $(0.1 \mu \mathrm{g})$, cultured in the absence or in the presence of $E_{2}\left(10^{-7} \mathrm{M}\right)$ for the indicated times, were assayed for telomerase activity by TRAP assay in the presence of an internal control (IC; $36 \mathrm{bp})$. As positive and negative controls, cell extracts $(0.1 \mu \mathrm{g})$ from telomerase-positive HeLa cells were assayed before and after heat inactivation (HeLa and HeLa H.I.), respectively. Panels relative to the internal control are shown at a lower detection exposure.

expression in all samples (Figure 1a), with a kinetic similar to that observed previously in the ovary epithelium (10). Following $\mathrm{E}_{2}$ treatment a reproducible increase in hTERT mRNA levels was also observed in fresh explants from $\mathrm{PCa}$ specimens (maximal stimulation ranging from 2.1- to 3.8-fold; Figure 1b) as well as in cell lines derived from PCa metastases (maximal stimulation ranging from 2.5- to 3-fold; Figure 1c).

Since the presence of steroid hormone responsive elements has been reported in the promoter region of the
RNA component of telomerase, hTR (32), we investigated using RT-PCR analysis whether hTR was also responsive to estrogen. No significant change in hTR mRNA levels was elicited in all samples by $\mathrm{E}_{2}$ treatment (Figure 1).

Telomerase activity, measured by TRAP, paralleled the induction profile of hTERT mRNA. $E_{2}$ treatment reversed telomerase silencing in telomerase-negative normal PrEC cells and in fresh explants from BPH (Figure 2a). Hormone-dependent stimulation of enzymatic activity, ranging from a three- to fivefold increase as compared with untreated samples, was also observed in telomerase-positive PCa specimens and cell lines (Figure $2, \mathrm{~b}$ and $\mathrm{c})$. These data indicate that in the prostate cell environment, there occurs a prompt estrogen modulation of telomerase activity, essentially accounted for by a significant induction of hTERT mRNA, suggesting a mechanism acting primarily at the transcriptional level.

$E_{2}$-dependent transcriptional activation of the $b T E R T$ promoter and in vivo recruitment of ER- $\alpha$ and ER- $\beta$ by chromatin immunoprecipitation assay. In a previous study (10) we demonstrated that expression of hTERT in ovary epithelium is controlled by estrogens, mainly at the level of transcription, through a specific interaction between the ER and an estrogen response element (ERE) located at position -949/-935 relative to the ATG within the hTERT 5 '-flanking region. Transient transfection studies showed that, in the presence of $\mathrm{E}_{2}$, overexpression of ER- $\alpha$ but not ER- $\beta$ remarkably increased $B T E R T$ promoter activity. We therefore asked whether ER signaling is involved in modulating hTERT transcriptional activity in the prostate environment. Extracts from prostate cells were first analyzed by Western blot to check the expression of endogenous steroid receptors. In agreement with recent reports $(12,33)$, we found that LNCaP and DU145 cells express predominantly ER- $\beta$ and are AR positive and negative, respectively (data not shown). Chimeric constructs containing the luciferase reporter fused to an hTERT promoter fragment with $5^{\prime}$ endpoint at -1009 relative to the ATG (encompassing the hTERT-ERE at -949/-935, either wild-type, p1009, or mutated, p1009Mut) were transfected alone or with expression vectors for ER- $\alpha$ and ER- $\beta$ in the presence or absence of $10^{-7} \mathrm{M} \mathrm{E}_{2}$, a saturating concentration at which both receptors demonstrate maximal transcriptional activity (34). In LNCaP and DU145 cells, estrogen treatment in the absence of exogenous receptors resulted in a significant increase (two- to fourfold) in the activity of the wild-type p1009 reporter (Figure 3a). Mutation of the hTERT-ERE (p1009Mut) essentially abrogated the estrogen responsiveness, thus emphasizing the importance and specificity of this motif. In agreement with previous result (10), no change in promoter activity was observed in ER-negative NIH3T3 cells. Upon estrogen stimulation, exogenously overexpressed ER- $\alpha$ caused a consistent upregulation of p1009 luciferase activity over the basal level in MCF-7 and LNCaP (fivefold) and DU145 (threefold) cells (Figure 3b). The variability in the magnitude of the reporter transcriptional activity upon $\mathrm{E}_{2}$ 
a

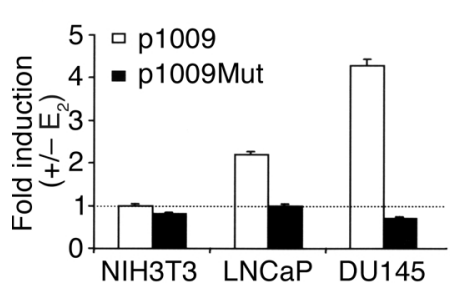

b

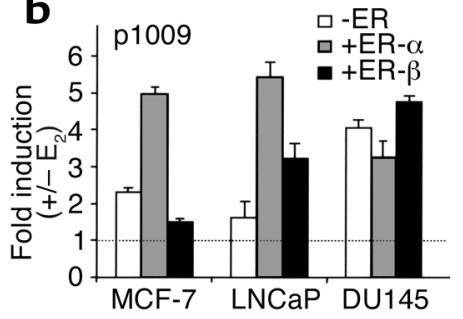

c

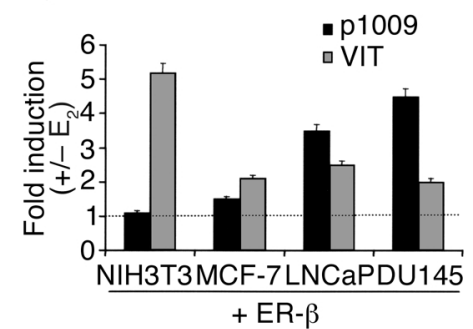

d<smiles></smiles>

$\mathrm{E}_{2}$

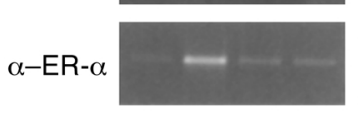

$\alpha-$ ER- $\beta$

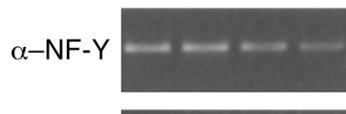

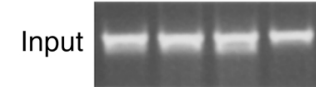

No $A b$
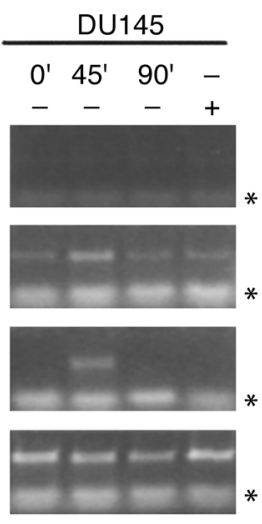

政

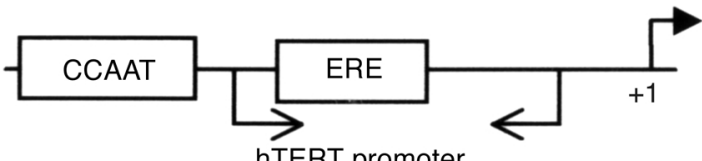

e

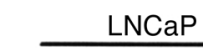

LNCaP
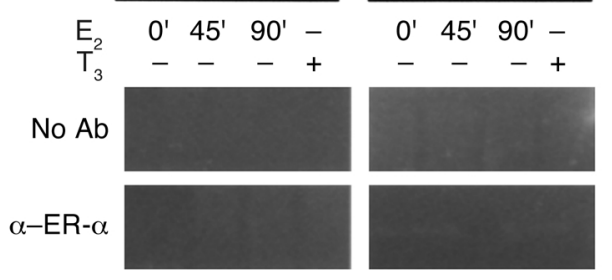

$\alpha-E R-\beta$
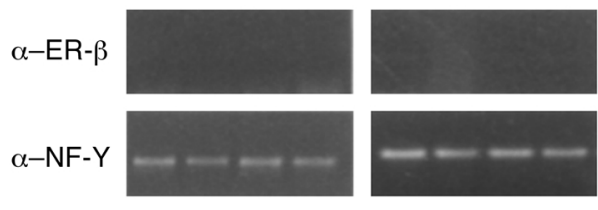

Input
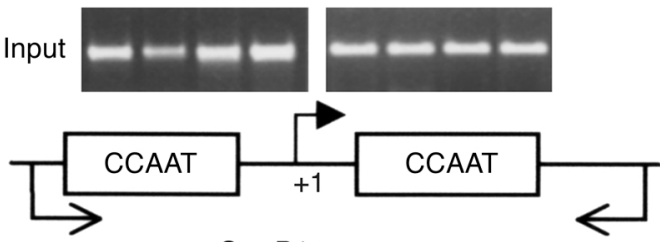

Cyc B1 promoter

\section{Figure 3}

Functional reporter and in vivo chromatin immunoprecipitation assays with hTERT promoter in PCa cells. Transient transfections with (a) p1009 or p1009Mut reporters containing wild-type or mutated proximal hTERT-ERE; (b) p1009 reporter alone (-ER), or in combination with expression vectors for ER- $\alpha(+E R-\alpha)$ or ER- $\beta(+E R-\beta)$; (c) p1009 or VIT reporters with expression vectors for ER- $\beta$ (+ER- $\beta)$ were performed. Values are expressed as fold of hormone induction, calculated as the ratio of normalized luciferase activity (light units per $\beta$-galactosidase units per minute per $\mu$ g of protein) in the presence or absence of $E_{2}\left(+/-E_{2}\right)$. Results represent the average $( \pm$ SEM) of six independent experiments, each performed in duplicate. Similar results were obtained with a longer hTERT located at $-949 /-935$ (proximal) and at $-2757 /-2738$ (distal) relative to the ATG (data not shown). Formaldehyde cross-linked chromatin from LNCaP and DU145 cells untreated ( $\left.0^{\prime}\right)$, or treated with $E_{2}\left(10^{-7} \mathrm{M}\right)$ for 45 and 90 minutes ( $45^{\prime}$ and $\left.90^{\prime}\right)$, or with triiodothyronine $\left(T_{3}, 10^{-7} \mathrm{M}\right)$ for 45 minutes ( $\left(^{+}\right)$was immunoprecipitated with Ab's to ER- $\alpha(\alpha-E R-\alpha)$, ER- $\beta$ ( $\alpha-E R-\beta), N F-Y(\alpha$ NF-Y), or in the absence of Ab's (no Ab) and analyzed by PCR with primers specific for the $h T E R T$ (d) and $C y c B 1$ (e) promoters. Input corresponds to nonimmunoprecipitated cross-linked chromatin. Primer position (arrows) on schematic promoters relative to the start site of transcription $(+1)$ and primer migration $\left({ }^{*}\right)$ are indicated.

treatment was not due to different intracellular levels of transfected ER- $\alpha$, as assessed by Western blot analysis (data not shown).

Notably, exogenously overexpressed ER- $\beta$ in the presence of $\mathrm{E}_{2}$ appeared to potentiate transactivation of the endogenous receptor in the PCa cells, whereas it was totally ineffective in modulating hTERT reporter activity in MCF-7 (Figure 3b) and NIH3T3 cells (Figure 3c) as well as in several other human nonprostate cell lines (WOO, MDA-MB-231, OVCA433; data not shown). To assess the functionality of exogenously expressed ER- $\beta$, the vitellogenin promoter (VIT), containing a perfect ERE, was used in parallel experiments (Figure 3c). Induction of this promoter over basal level by ER- $\beta$ in the presence of $E_{2}$ was indeed observed in the nonprostate cell lines, thus suggesting that hTERT promoter sensitivity to ER- $\beta$ is limited to the prostate environment. These findings are remarkable since in a nonprostate cell background the estrogen responsiveness of the $b T E R T$ promoter is essentially mediated by ER- $\alpha(10,31)$.

To elucidate DNA binding activity of both ERs to the hTERT estrogen responsive element we investigated the in vivo interaction of ligand-activated ER- $\alpha$ or ER- $\beta$ to the hTERT promoter in LNCaP and DU145 cells before and after estrogen treatment by chromatincrosslinked immunoprecipitation (ChIP) (29). Cells were grown in the absence of estrogen for 5 days, followed by treatment with $\mathrm{E}_{2}\left(10^{-7} \mathrm{M}\right)$ for 45 and 90 minutes (35). After formaldehyde cross-linking and precipitation of the chromatin with ER- $\alpha$ and ER- $\beta$ Ab's, the precipitated DNA was subjected to PCR amplification using specific primers spanning the endogenous proximal hTERT-ERE (-949/-935). Treatment with $\mathrm{E}_{2}$ resulted in recruitment of both ER- $\alpha$ and ER- $\beta$ onto 
a



b

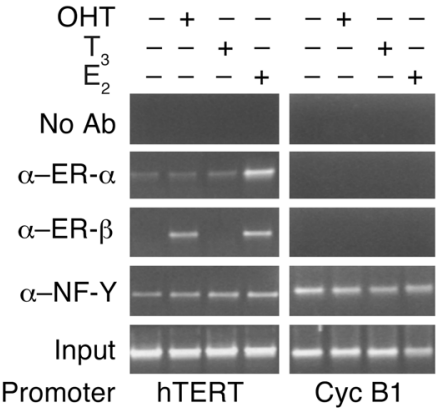

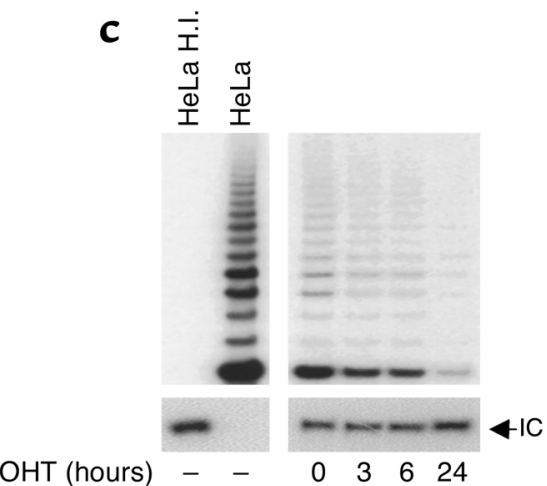

\section{Figure 4}

$\mathrm{E}_{2}$ and OHT differentially modulate ER signaling on the hTERT promoter and on telomerase activity in LNCaP cells. (a) Cotransfections of p1009 reporter alone (-ER) or in combination with expression vectors for ER- $\alpha(+E R-\alpha)$ or ER- $\beta(+E R-\beta)$ were performed with or without ligand $\left(E_{2}\right.$ or $\left.\mathrm{OHT}\right)$. Results represent the average $( \pm \mathrm{SEM})$ of four independent experiments, each performed in duplicate, and values are expressed as fold induction (+/- ligand), as described in the legend to Figure 3. (b) ChIP assay was performed in LNCaP untreated (-) or treated $(+)$ with $\mathrm{E}_{2}, \mathrm{OHT}$, or $\mathrm{T}_{3}$, as described in the legend to Figure 3. (c) Extracts $(0.25 \mu \mathrm{g})$ from cells treated with $\mathrm{OHT}$ for $0,3,6$, and 24 hours were assayed for telomerase activity by TRAP.

the $b T E R T$ promoter (Figure $3 \mathrm{~d}$ ). The ER- $\alpha$ signal was already detectable in the absence of the hormone, increased after 45 minutes upon $\mathrm{E}_{2}$ addition, and returned to baseline by 90 minutes, thus resembling the dynamic profile described for ER- $\alpha$ association with three well-characterized estrogen target genes (35). Similarly, ER- $\beta$ was recruited onto the hTERT promoter by 45 minutes of treatment and dissociated by 90 minutes. Treatment of cells with an unrelated ligand, the thyroid hormone $T_{3}$, did not induce an association of ER- $\alpha$ or ER- $\beta$ with the hTERT promoter. Since we could not use sequences upstream of the proximal hTERT-ERE as negative "control promoter", given the presence of the distal hTERT-ERE (-2757/-2738) within the size range of DNA fragments generated by sonication, the sequence of the cell cycle-dependent human $\mathrm{Cyc} \mathrm{B} 1$ promoter was chosen. This promoter has been shown to specifically recruit the CCAAT-binding factor NF-Y in the ChIP assay (30). As shown in Figure 3e, no amplification was detected in the ERE-negative cyclin B1 promoter when using ER- $\alpha-$ and ER- $\beta$-specific $A b$ 's, while a positive signal was elicited in the NF-Y immunoprecipitates as expected. It is of interest that with the hTERT promoter we detected a specific signal using the NF-Y Ab, regardless of hormonal treatment, probably resulting from direct binding of this transcription factor to CCAAT boxes flanking the hTERTERE region. As a negative control, the chromatin was immunoprecipitated either without (no $\mathrm{Ab}$ ) or with a $\beta$-galactosidase $\mathrm{Ab}$ (data not shown). In these immunoprecipitates no amplification of hTERT or $\mathrm{CycB} 1$ sequences could be detected.

To rule out a nonspecific association between ERs and the hTERT promoter, we used several polyclonal $A b$ 's and $m A b$ 's directed toward different receptor epitopes in the ChIP assay. Four different Ab's for ER- $\alpha$ and two for ER- $\beta$ gave almost identical patterns of association with the hTERT promoter.
These data clearly indicate a specific estrogendependent recruitment of both ER- $\alpha$ and ER- $\beta$ to the hTERT promoter in the prostate environment.

$E_{2}$ and tamoxifen as modulators of ER signaling on bTERT promoter and telomerase activity. Although ER- $\alpha$ and ER- $\beta$ share similar binding domains and both bind EREs, they exhibit distinct transactivation properties in different cell backgrounds in response to classical agonists, such as $\mathrm{E}_{2}$, or selective ER modulators (SERMs), such as tamoxifen or raloxifen, which function as antagonists but also as partial ER- $\alpha$ agonists $(20,36)$. To elucidate the specific role of ER- $\alpha$ and ER- $\beta$ in the transcriptional regulation of the bTERT promoter, functional experiments were carried out in LNCaP cells transfected with the p1009 construct and treated or not with $\mathrm{E}_{2}$ or OHT $\left(10^{-7} \mathrm{M}\right)$ in the presence of the endogenous receptor(s) or after overexpression of ERs. Treatment with $\mathrm{E}_{2}$, as previously observed, increased p1009 transcriptional activity over the basal level, whereas treatment with OHT did not elicit a transcriptional response (Figure 4a). Upon overexpression of ER- $\alpha, E_{2}$ and OHT induced reporter activity with similar potency (five and sixfold, respectively). It is interesting that upon ER- $\beta$ overexpression, while $E_{2}$ induced the hTERT promoter, OHT repressed hTERT transcriptional activity below basal levels.

These findings support previous observations indicating that in different cell contexts SERMs act as pure antagonists by way of ER- $\beta$ when bound to EREs, while they are capable of potentiating ER- $\alpha$ transactivation properties $(20,36)$. The findings also indicate that ER- $\alpha$ and ER- $\beta$ can differentially contribute to the transcriptional regulation of the hTERT promoter in the prostate environment.

Because this opposite response substantially reflects ER conformational changes specifically induced by the interaction with structurally distinct 
ligands $(20,37)$, we asked whether OHT treatment could affect endogenous $\mathrm{ER}$ in vivo recruitment onto the hTERT promoter. ChIP analysis showed that, as observed with $\mathrm{E}_{2}$, OHT treatment recruited ER- $\beta$ occupancy of the hTERT promoter in LNCaP (Figure 4b) and DU145 cells (data not shown). In contrast, no OHT-dependent induction of ER- $\alpha$ was elicited, indicating that OHT-mediated differential recruitment of ER- $\alpha$ and ER- $\beta$ ultimately modulates hTERT gene transcription.

We further investigated the effects of differential agonist/antagonist treatment on telomerase activity by TRAP. As predicted from functional and ChIP experiments, addition of OHT significantly inhibited the enzymatic activity in LNCaP (Figure 4c) and in DU145 cells (data not shown), confirming the negative regulation of telomerase activity through OHTmediated ER signaling.

This finding discloses a novel role of ERs in modulating telomerase in the prostate environment and allows, potentially, prediction of the biological response to hormone-based therapeutic approaches. Effects of the selective aromatase in bibitor on telomerase activity. It is traditionally assumed that PCa is strictly androgen dependent in its growth and malignant progression. Our results, pointing to a specific role of ERs in regulating telomerase activity in normal and neoplastic prostate epithelial cells, indicate a potential role of estrogens in prostate carcinogenesis. In this context, local conversion of androgens to estrogens by aromatase (38) may acquire pathophysiological significance by exposing prostate epithelial cells to amounts of estrogens higher than those circulating in blood. We therefore used the nonsteroidal aromatase inhibitor, letrozole, to strengthen the role of ER pathways in governing hTERT activation in PCa cells. Nuclear extracts were prepared from LNCaP cells cultured in the presence or absence of $\mathrm{E}_{2}\left(10^{-7} \mathrm{M}\right)$ or $\mathrm{T}\left(10^{-7} \mathrm{M}\right)$ with or without letrozole $(1 \mu \mathrm{M})$. Mobility-shift analysis was performed using oligonucleotides spanning the proximal hTERT-ERE (Figure $5 a)$. A protein complex was elicited upon incubation of nuclear extracts in the unstimulated condition (lane 2). In the presence of $\mathrm{E}_{2}$ a slow-migrating band was stabilized (lane 4, arrow). In all conditions protein complexes were promptly competed out by cold-specific oligonucleotide, but not mutant oligonucleotide (data not shown). Addition of letrozole did not affect binding of the endogenous ER to hTERT-ERE in untreated (compare lanes 2 and 3 ) or $E_{2}$-treated (compare lanes 4 and 5) cells. It is of interest that treatment with $\mathrm{T}$ yielded a binding pattern similar to that obtained with $\mathrm{E}_{2}$ treatment (compare lanes 4 and 6). In the presence of letrozole, however, formation of this specific binding pattern was prevented (compare lane 6 and 9). To ascertain whether the binding pattern detected upon T treatment (lane 6) was, in fact, produced by interaction of $E_{2}$-activated $E R$ to hTERTERE (upon conversion of T to $E_{2}$ ), ER- $\beta$ or AR Ab's were added to the binding mixture. Addition of anti-ER- $\beta \mathrm{Ab}$ (lane 8), but not of anti-AR Ab (lane 7), abrogated the specific interaction.

To investigate whether the impairment of in vitro binding in LNCaP cells following letrozole addition could correlate with modifications in telomerase activity, we performed TRAP assays with LNCaP cells upon treatment with $\mathrm{T}$ or the nonaromatizable synthetic androgen R1881 $\left(10^{-8} \mathrm{M}\right)$ in the presence or absence of the aromatase inhibitor letrozole (Figure $5 \mathrm{~b}$ ). No significant modifications were observed between treatment with R1881 or combination of R1881 plus letrozole, whereas combination of $\mathrm{T}$ and letrozole significantly inhibited TRAP activity. These results indicate that in PCa cells conversion of $\mathrm{T}$ to estrogens may favor telomerase activity, allowing speculation on the use of aromatase inhibitors as potential drug candidates in the treatment of $\mathrm{PCa}$.

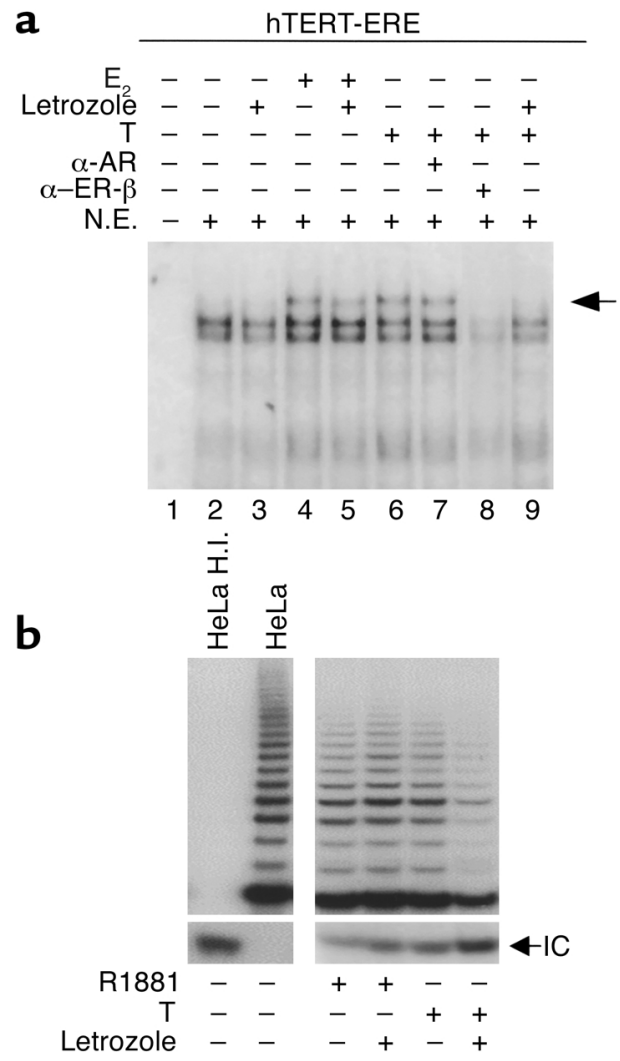

Figure 5

Effect of the selective aromatase inhibitor letrozole on the interaction between ERs and the hTERT-ERE and on telomerase activity. (a) LNCaP cells were cultured for 16 hours in the absence (lanes 2 and 3 ) or the presence of $\mathrm{E}_{2}\left(10^{-7} \mathrm{M}\right.$, lanes 4 and 5$)$ or $\mathrm{T}\left(10^{-7} \mathrm{M}\right)$ (lanes 6-9) with (lanes 3, 5, and 9) or without the nonsteroidal aromatase inhibitor letrozole $(1 \mu \mathrm{M})$ added 1 hour before hormone addition. Nuclear extracts $(5 \mu \mathrm{g})$ were then prepared and incubated with a ${ }^{32}$ P-labeled oligonucleotide encompassing the proximal hTERT-ERE. Reaction in the presence of anti-AR ( $\alpha A R)$ or anti-ER- $\beta(\alpha E R \beta)$ Ab's are shown in lanes 7 and 8 , respectively. Lane 1 corresponds to probe incubated without nuclear extracts. Migration of a slower migrating complex is indicated (arrow). (b) Extracts $(0.1 \mu \mathrm{g})$ from LNCaP cells treated for 4 hours with R1881 $\left(10^{-8} \mathrm{M}\right)$ or $\mathrm{T}\left(10^{-7} \mathrm{M}\right)$ with or without the nonsteroidal aromatase inhibitor letrozole $(1 \mu \mathrm{M})$ added 1 hour before ligand addition were assayed for telomerase activity by TRAP. 


\section{Discussion}

PCa is the second leading cause of cancer death in men (39). Despite efforts made toward early detection of these lesions, some of them progress into invasive cancers, for which effective cure is not currently available. One of the earliest molecular alterations in PCa is telomerase reactivation $(8,9)$, which is thought to play a key role during tumor progression. Sex steroid receptors are well-recognized hormonal effectors in prostate tumorigenesis (7). In the present study we propose an essential involvement of ER signaling in the malignant conversion of human prostate epithelium through reactivation and/or modulation of telomerase. We also provide evidence in favor of a relevant role of ERs as therapeutic targets in PCa. A prompt induction of hTERT mRNA and of telomerase activity is detected in normal and transformed human prostate epithelial cells upon $\mathrm{E}_{2}$ treatment, suggesting a mechanism acting primarily at the transcription level. It is of interest that functional studies show that in the prostate cell environment, $E_{2}$ stimulation of ER- $\beta$, in addition to that of ER- $\alpha$, significantly increased hTERT promoter activity. Results from ChIP assays showing specific and $E_{2}$-dependent in vivo recruitment of ER- $\beta$ and ER- $\alpha$ onto the hTERT promoter substantiate a direct role of ERs in controlling expression of the catalytic subunit of the enzyme whose activity allows unlimited cell proliferation.

Our hypothesis favoring an etiopathogenetic role of ERs in prostate tumorigenesis may appear at odds with the well-known androgen dependency of prostate tumors. However, the age-dependent decline of androgens-to-estrogens ratio has been already suggested as a pathogenetic factor for prostate tumor development (7). In addition, local conversion of androgens to estrogens by aromatase may provide significant amounts of intracellular estrogens, leading to activation of endogenous ERs. Although molecular and cellular mechanisms responsible for estrogen formation in the prostate, and their physiological and clinical relevance, are currently under investigation, it should be emphasized that in breast cancer aromatase inhibitors have been proposed as second-line drugs in the hormonal therapy of this disease to achieve a complete estrogen deprivation (40). Indeed, in our study, the combination of $\mathrm{T}$ and letrozole resulted in a consistent inhibition of telomerase activity. In this regard we hypothesize that the androgen effects on the prostate gland may be mediated, at least in part, by ERs rather than by ARs, following aromatization of androgen to estrogen. The recent identification of the $\mathrm{T}$ metabolite $5 \alpha$-androstane$3 \beta, 17 \beta$-diol as a specific ligand of ER- $\beta$ in rat ventral prostate (15) further supports our hypothesis of a relevant role of ER signaling in prostate pathophysiology.

Our data highlight two critical issues in the clinical oncology of the prostate gland: the possibility of regulating telomerase activity in human prostate malignant epithelium through agonist/antagonist modulation of ER function and the relative expression of both ERs as reference criteria in directing potential endocrine therapeutical approaches. Indeed, since interaction between the epithelial and stromal components seems to be essential for normal prostate growth and development, the compartmentalization of the two ERs ER- $\alpha$ exclusively in the stroma and ER- $\beta$ mostly in the epithelial compartment $(7,14)$ of normal and malignant human prostate tissue - can account for a potential role of each receptor in perturbing such complex stromal-epithelial interaction (41).

Although a recent study (14) on a large cohort of PCa patients reported a prevalent decrease in ER- $\beta$ expression in most cases, it also supported a role for ER- $\beta$ in tumor progression by demonstrating that those cases that retained ER- $\beta$ had a more aggressive phenotype and poorer prognosis. It would be of interest to measure telomerase activity and telomere length in this group of tumors, since persistence of ER- $\beta$ expression and subsequent telomerase induction may contribute to extending cell life span, thus favoring genetic events leading to acquisition of the more malignant phenotype. Our data demonstrating estrogen-dependent telomerase activation in normal and transformed prostate epithelial cells strongly favor a facilitating role of telomerase in tumor progression. Similar conclusions have been drawn from studies with telomerase-deficient mice, showing that, although telomere dysfunction may facilitate carcinogenesis (42), lack of telomerase is associated with resistance to cancer (43-46).

An additional outcome of our study is the possibility of negatively regulating the $b T E R T$ promoter and telomerase activities in PCa cells by OHT treatment. It is of interest that our ChiP analysis showed a specific OHTdependent recruitment of ER- $\beta$, but not ER- $\alpha$, on the hTERT promoter. This differential occupancy may be accounted for by an active involvement of the OHT-ER$\beta$ complex in gene repression, probably through interaction with cofactor(s) highly involved in nuclear hormone receptor signaling (47). The absence of OHT-mediated ER- $\alpha$ recruitment, although not completely ruling out ER- $\alpha$ involvement, allows us to hypothesize that, in the prostate, tamoxifen exerts its antagonist function essentially through the ER- $\beta$ pathway. This view mirrors data from Shang et al. (35), showing, in transformed breast epithelium (a tissue in which the antagonistic action of tamoxifen is well characterized), a specific tamoxifenmediated ER- $\alpha$ recruitment onto estrogen target gene promoters. The antiproliferative effects of tamoxifen in DU145 cells and the reversal of this process by treatment with ER- $\beta$ antisense oligonucleotides (12), strongly support our conclusions. An antiestrogen treatment of ER$\beta$-positive prostate tumors is also envisaged by Horvath et al. in their recent study (14).

Our results, demonstrating a tight control of telomerase activity by ERs in human normal and malignant prostate epithelial cells, suggest the possibility of predicting the biological response to hormone-based therapies, commonly adopted in the management of PCa, with relevant implications in clinical oncology. 


\section{Acknowledgments}

This work was supported by grants from Associazione Italiana Ricerca sul Cancro (AIRC), Progetto Strategico "Oncologia" MIUR-CNR, MIUR, and Ministero della Salute. We thank Aymone Gurtner and Giulia Piaggio for sharing their expertise in chromatin immunoprecipitation and Silvia Bacchetti and Carlo Gaetano for helpful discussion and critical reading of the manuscript. This work is dedicated to the memory of Franco Tató.

1. Artandi, S.E., and DePinho, R.A. 2000. A critical role for telomeres in suppressing and facilitating carcinogenesis. Curr. Opin. Gen. Dev. 10:9-46.

2. Hanahan, D., and Weinberg, R.A. 2000. The hallmarks of cancer. Cell. 100:57-70.

3. Bodnar, A.G., et al. 1998. Extension of life-span by introduction of telomerase into normal human cells. Science. 279:349-352.

4. Vaziri, H., and Benchimol, S. 1998. Reconstitution of telomerase activity in normal human cells leads to elongation of telomeres and extended replicative life span. Curr. Biol. 8:279-282.

5. Hahn, W.C., et al. 1999. Inhibition of telomerase limits the growth of human cancer cells. Nat. Med. 5:1164-1170.

6. Shay, J.W., and Bacchetti, S. 1997. A survey of telomerase activity in human cancer. Eur. J. Cancer. 33:787-791.

7. Abate-Shen, C., and Shen, M. 2000. Molecular genetics of prostate cancer. Genes Dev. 14:2410-2434.

8. Sommerfeld, H.J., et al. 1996. Telomerase activity: a prevalent marker of malignant human prostate tissue. Cancer Res. 56:218-222.

9. Zhang, W., Kapusta, L.R., Slingerland, J.M., and Klotz, L.H. 1998. Telomerase activity in prostate cancer, prostatic intraepithelial neoplasia, and benign prostatic epithelium. Cancer Res. 58:619-621.

10. Misiti, S., et al. 2000. Induction of hTERT expression and telomerase activity by estrogens in human ovary epithelium cells. Mol. Cell. Biol. 20:3764-3771.

11. Chang, W.Y., and Prins, G.S. 1999. Estrogen receptor- $\beta$ : implications for the prostate gland. Prostate. 40:115-124.

12. Lau, K.M., LaSpina, M., Long, J., and Ho, S.M. 2000. Expression of estrogen receptor (ER)- $\alpha$ and ER- $\beta$ in normal and malignant prostatic epithelial cells: regulation by methylation and involvement in growth regulation. Cancer Res. 60:3175-3182.

13. Li, L.C., et al. 2000. Frequent methylation of estrogen receptor in prostate cancer: correlation with tumor progression. Cancer Res. 60:702-706.

14 . Horvath, L.G., et al. 2001. Frequent loss of estrogen receptor- $\beta$ expression in prostate cancer. Cancer Res. 61:5331-5335.

15. Weihua, Z., et al. 2001. A role for estrogen receptor $\beta$ in the regulation of growth of the ventral prostate. Proc. Natl. Acad. Sci. USA. 98:6330-6335.

16. Prehn, R.T. 1999. On the prevention and therapy of the prostate cancer by androgen administration. Cancer Res. 59:4161-4164.

17. Mangan, F.R., Neal, G.E., and William, D.C. 1967. The effects of diethylstilboestrol and castration on the nucleic acid and protein metabolism of rat prostate gland. Biochem. J. 104:1075-1081.

18. Bonkhoff, H., Fixemer, T., Hunsicker, I., and Remberger, K. 1999. Estrogen receptor expression in prostate cancer and premalignant prostatic lesions. Am. J. Pathol. 155:641-647.

19. Couse, J.F., Curtis Hewitt, S., and Korach, K.S. 2000. Receptor null mice reveal contrasting roles for estrogen receptor $\alpha$ and $\beta$ in reproductive tissues. J. Steroid Biochem. Mol. Biol. 74:287-296.

20. Pettersson, K., and Gustafsson J.A. 2001. Role of estrogen receptor beta in estrogen action. Annu. Rev. Physiol. 63:165-192.

21. Leav, I., et al. 2001. Comparative studies of the estrogen receptors $\beta$ and $\alpha$ and the androgen receptor in normal human prostate glands, dysplasia, and in primary and metastatic carcinoma. Am. J. Pathol. 159:79-92.

22. Latil, A., et al. 2001. Evaluation of androgen, estrogen (ER $\alpha$ and $E R \beta)$, and progesterone receptor expression in human prostate cancer by realtime quantitative reverse transcription-polymerase chain reaction assay. Cancer Res. 6:1919-1926.
23. Pasquali, D., et al. 2001. Loss of estrogen receptor $\beta$ expression in malig nant human prostate cells in primary cultures and in prostate cancer tissues. J. Clin. Endocrinol. Metab. 86:2051-2055.

24. Carruba, G., et al. 1996. Multiple estrogen function in human prostate cancer cells. Ann. NY Acad. Sci. 784:70-84.

25. Liu, H., et al. 1997. Monoclonal antibodies to the extracellular domain of prostate-specific membrane antigen also react with tumor vascular endothelium. Cancer Res. 57:3629-3634.

26. Horwitz, K.B., and McGuire, W.L. 1987. Estrogen control of progesterone receptor in human breast cancer. J. Biol. Chem. 266:1008-1013.

27. Ulaner, G.A., Hu, J.F., Vu, T.H., Giudice, L.C., and Hoffman, R. 1998. Telomerase activity in human development is regulated by human telomerase reverse transcription and by alternative splicing of hTERT transcript. Cancer Res. 58:4168-4172.

28. Kim, N.W., and Wu, F. 1997. Advances in quantification and characterization of telomerase activity by the telomeric repeat amplification protocol (TRAP). Nucleic Acids Res. 25:2595-2597.

29. Boyd, K.E., Wells, J., Gutman, J., Bartely, S.M., and Farnham, P.J. 1998. C-Myc target gene specificity determined by a post-DNA-binding mechanism. Proc. Natl. Acad. Sci. USA. 95:13887-13892.

30. Sciortino, S., et al. 2001. The cyclin B1 gene is actively transcribed during mitosis in HeLa cells. EMBO Rep. 21:1018-1023.

31. Kyo, S., et al. 1999. Estrogen activates telomerase. Cancer Res. 59:5917-5921.

32. Zhao, J.Q., et al. 1998. Cloning and characterization of human and mouse telomerase RNA gene promotor sequences. Oncogene. 16:1345-1350.

33. Ye, Q., Chung, L.W.K., Cinar, B., Li, S., and Zhau, H.E. 2000. Identification and characterization of estrogen receptor variants in prostate cancer cell lines. J. Steroid Biochem. Mol. Biol. 75:21-31.

34. Hall, J.M., and McDonnell, D.P. 1999. The estrogen receptor $\beta$-isoform $(\operatorname{Er} \beta)$ of the human estrogen receptor modulates Er $\alpha$ transcriptional activity and is a key regulator of the cellular response to estrogens and antiestrogens. Endocrinology. 140:5566-5578.

35. Shang, Y., Hu, X., DiRenzo, J., Lazar, M.A., and Brown, M. 2000. Cofactor dynamics and sufficiency in estrogen receptor-regulated transcription. Cell. 103:843-852.

36. McInerney, E.M., Weis, K.E., Sun, J., Mosselman, S., and Katzenellenbogen, B.S. 1998. Transcription activation by the human estrogen receptor subtype $\beta$ (ER $\beta)$ studied with $E R \beta$ and $E R \alpha$ receptor chimeras. Endocrinology. 139:4513-4522.

37. Shiau, A.K., et al. 1998. The structural basis of estrogen receptor/coactivator recognition and the antagonism of this interaction by tamoxifen. Cell. 95:927-937.

38. Bhatnagar, A.S., Brodie, A.M., Long, B.J., Evans, D.B., and Miller, W.R. 2001. Intracellular aromatase and its relevance to the pharmacological efficacy of aromatase inhibitors. J. Steroid Biochem. Mol. Biol. 76:199-202.

39. Coffey, D.S. 1993. Prostate cancer. An overview of an increasing dilemma. Cancer. 71:880-886.

40. Buzdar, A.U. 2001. Endocrine therapy in the treatment of metastatic breast cancer. Semin. Oncol. 28:291-304.

41. Matrisian, L.M., Cunha, G.R., and Mohla, S. 2001. Epithelial-stromal interactions and tumor progression: meeting summary and future directions. Cancer Res. 61:3844-3846.

42. Hackett, J.A., and Greider, C.W. 2002. Balancing instability: dual roles for telomerase and telomere dysfunction in tumorigenesis. Oncogene. 21:619-626.

43. Greenberg, R.A., et al. 1999. Short dysfunctional telomeres impair tumorigenesis in the INK4a $\mathrm{a}^{\Delta 2 / 3}$ cancer-prone mouse. Cell. 97:515-525.

44. Chin, L., et al. 1999. p53 deficiency rescues the adverse effects of telomere loss and cooperates with telomere dysfunction to accelerate carcinogenesis. Cell. 97:527-538.

45. Gonzàlez-Suàrez, E., Samper, E., Flores, J.M., and Blasco, M.A. 2000. Telomerase-deficient mice with short telomeres are resistant to skin tumorigenesis. Nat. Genet. 26:114-117.

46. Rudolph, K.L., Millard, M., Bosenberg, M.W., and DePinho, R.A. 2001. Telomere dysfunction and evolution of intestinal carcinoma in mice and humans. Nat. Genet. 28:155-159.

47. McKenna, N.J., Rainer, B., and O'Malley, B.W. 1999. Nuclear receptor coregulators: cellular and molecular biology. Endocri. Rev. 20:321-344. 